\title{
Nuclear Lattice Simulations with EFT
}

\author{
Dean Lee ${ }^{\mathrm{a}}$

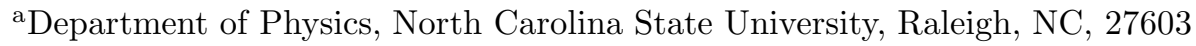

This proceedings article is a summary of results from work done in collaboration with Bugra Borasoy and Thomas Schaefer. We study nuclear and neutron matter by combining chiral effective field theory with nonperturbative lattice methods. We present results for hot neutron matter at temperatures 20 to $40 \mathrm{MeV}$ and densities below twice nuclear matter density.

\section{Introduction}

Weinberg extended effective field theory methods to the nucleon-nucleon interaction [1, and over the last several years effective field theory methods have been applied successfully to the two and three-nucleon system. Our aim and the goal of the Nuclear Lattice Collaboration as a whole [2] [3] is to extend effective field theory methods to the nuclear many-body problem. For this purpose we investigate the many-body physics of lowenergy nucleons and pions on the lattice. Our starting point is the same as that of Weinberg. We begin with the most general local Lagrangian involving pions and low-energy nucleons consistent with translational invariance, isospin symmetry, and spontaneously broken chiral symmetry. This yields an infinite set of possible interaction terms with increasing numbers of derivatives and/or nucleon fields. Degrees of freedom associated with anti-nucleons, heavier mesons such as the $\rho$, and heavier baryons such as the $\Delta$, are integrated out. The contribution of these particles appear as coefficients of local terms in our pion-nucleon Lagrangian. We also integrate out nucleons with momenta greater than $\pi a^{-1}$, where $a$ is the lattice spacing.

The operator coefficients in our effective Lagrangian are determined by fitting to experimentally-measured few-body nucleon scattering data at zero temperature. The dependence on the lattice spacing is described by the renormalization group and can absorbed by renormalizing operator coefficients. In this way we construct a realistic simulation of many-body nuclear phenomena with no free parameters. In our discussion we present results for hot neutron matter at temperatures 20 to $40 \mathrm{MeV}$ and densities below twice nuclear matter density. This proceedings article is a summary of results from work done in collaboration with Buḡra Borasoy and Thomas Schaefer [4].

\section{Non-perturbative effective field theory}

Effective field theory provides a systematic method to compute physical observables order by order in the small parameter $Q / \Lambda_{\chi}$, where $\Lambda_{\chi}$ is the chiral symmetry breaking scale and $Q=\left(q, m_{\pi}, \ldots\right)$. Here, $q$ is a small external momentum and $m_{\pi}$ is the mass of the pion. The simplest processes are those that involve only pions and external fields. In this case the effective field theory is perturbative. At any order in $Q$ there are only a finite number of diagrams that have to be included. At lowest order these are tree diagrams with the leading order interaction. At higher order, diagrams with more loops or higher order terms in the interaction have to be taken into account.

Weinberg showed [1] [5] that the simple diagrammatic expansion for nucleon-nucleon scattering is spoiled by infrared divergences. $\mathrm{He}$ suggested performing an expansion of the twoparticle irreducible kernel and then iterating the kernel to all orders to produce the scattering Green's function. It was later pointed out that a possible difficulty arises because at any order 
in $Q$ an infinite number of diagrams is summed, and it is not clear that all the cutoff dependence at that order can be absorbed into counterterms that are present at that order 6]. This problem does indeed arise if one considers nucleon-nucleon scattering in the ${ }^{1} S_{0}$ channel [7, but in practice the cutoff dependence appears to be very weak 8.

In this work we go one step further and consider the nuclear many-body problem. We expand the terms in our action order by order, $S=S_{0}+S_{1}+$ $S_{2}+\cdots$. At order $k$ in the chiral expansion, we calculate observables by evaluating the functional integral

$$
\frac{\int D N D \bar{N} D \pi G(\bar{N}, N, \pi) e^{-\left[S_{0}+\cdots+S_{k}\right]}}{\int D N D \bar{N} D \pi e^{-\left[S_{0}+\cdots+S_{k}\right]}} .
$$

We will refer to this approach as nonperturbative effective field theory. The interactions at chiral order $k$ or less are iterated to arbitrary loop order. The functional integral is computed non-perturbatively by putting the pion and nucleon fields on the lattice and using Monte Carlo sampling. Since the number of diagrams at a given chiral order grows exponentially with the number of nucleons, a non-perturbative technique such as this is needed for systems with more than just a few nucleons.

Computing the path integral corresponds to summing an infinite set of diagrams. As in the case of iterating the two-particle irreducible kernel to determine the full two-nucleon Green's function, it is not clear that the cutoff dependence at a given order in the low energy expansion can be absorbed into a finite number of coefficients in the action. In practice we will therefore restrict ourselves to lattice cutoffs that satisfy $\pi a^{-1}<\Lambda_{\chi}$.

\section{Lowest order interactions}

We let $N$ represent the nucleon fields. We use $\tau_{i}$ to represent Pauli matrices acting in isospin space, and we use $\vec{\sigma}$ to represent Pauli matrices acting in spin space. Pion fields are notated as $\pi_{i}$. We denote the pion decay constant as $F_{\pi}^{\text {phys }} \approx$ $183 \mathrm{MeV}$ and let $D=1+\pi_{i}^{2} / F_{\pi}^{2}$. The lowest order Lagrange density for low-energy pions and nucleons is given by [?

$$
\begin{aligned}
\mathcal{L}^{(0)} & =-\frac{1}{2} D^{-2}\left[\left(\vec{\nabla} \pi_{i}\right)^{2}-\dot{\pi}_{i}^{2}\right]-\frac{1}{2} D^{-1} m_{\pi}^{2} \pi_{i}^{2} \\
& +\bar{N}\left[i \partial_{0}-\left(m_{N}-\mu\right)\right] N \\
& -D^{-1} F_{\pi}^{-1} g_{A} \bar{N}\left[\tau_{i} \vec{\sigma} \cdot \vec{\nabla} \pi_{i}\right] N \\
& -D^{-1} F_{\pi}^{-2} \bar{N}\left[\epsilon_{i j k} \tau_{i} \pi_{j} \dot{\pi}_{k}\right] N \\
& -\frac{C_{S}}{2} \bar{N} N \bar{N} N-\frac{C_{T}}{2} \bar{N} \vec{\sigma} N \cdot \bar{N} \vec{\sigma} N
\end{aligned}
$$

$g_{A}$ is the nucleon axial coupling, and $\epsilon_{i j k}$ is the Levi-Civita symbol. The chemical potential $\mu$ controls the nucleon density and $\mu$ will be set very close to $m_{N}$. At next order we have terms

$$
\mathcal{L}^{(1)}=\frac{1}{2 m_{N}} \bar{N} \vec{\nabla}^{2} N+\ldots
$$

We will include this kinetic energy term from $\mathcal{L}^{(1)}$ in our lowest-order Lagrange density so that we get the usual free nucleon propagator. In this study we limit ourselves to the interactions of neutrons and neutral pions.

\section{Lattice Schrödinger equation and phase shifts}

We adjust $C$, the coefficient of the $\bar{N} N \bar{N} N$ contact interaction, so that the $N N$ s-wave scattering length matches the experimental value (see for example [10]). In order to calculate the phase shifts, we will solve the lattice Schrödinger equation for the two-neutron system and observe the asymptotic form of the scattering wavefunctions.

With the lowest order two-nucleon potential we can construct a matrix representation for the Hamiltonian in the two-neutron sector and solve the time-independent lattice Schrödinger equation. At this stage one could implement Lüscher's formula for measuring phase shifts in a cubical periodic box 11] 12. However in our case we can construct the eigenvectors explicitly using Lanczos iteration, and so we find it more straightforward and accurate to read the phase shifts directly from the asymptotic forms of the s-wave scattering states. We use the lattice Schrödinger technique to tune the coupling $C$ to reproduce the large scattering length that is observed in nature. 


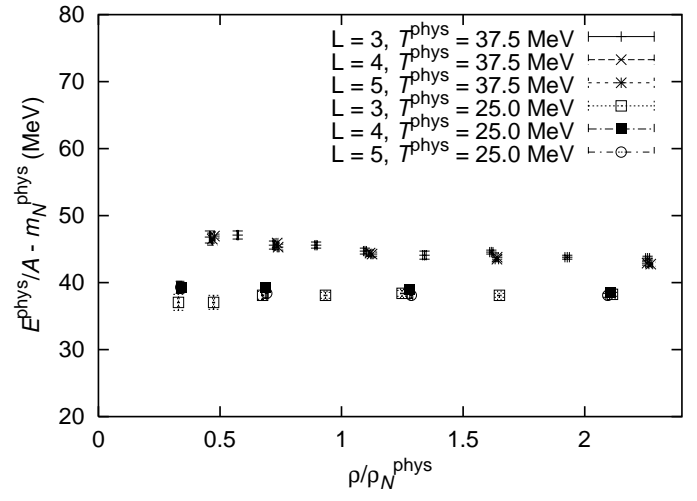

Figure 1. Energy per neutron in $\mathrm{MeV}$ for temperatures $25 \mathrm{MeV}$ and $37.5 \mathrm{MeV}$ and different lattice volumes. The inverse lattice spacing is $a^{-1}=150$ $\mathrm{MeV}$ and $\alpha_{t}=1.0$.

\section{Results}

We have generated simulation results for $a^{-1}=$ $150 \mathrm{MeV} ; \alpha_{t}=\frac{a_{t}}{a}=1.0$; temperatures $T^{p h y s}=$ $25.0 \mathrm{MeV}$ and $37.5 \mathrm{MeV}$; and lattice sizes $3^{3}, 4^{3}$, and $5^{3}$. Half-filling at this lattice spacing occurs at $\rho=2.64 \rho_{N}^{\text {phys }}$, where $\rho_{N}^{\text {phys }}$ is the normal nuclear density of about 0.17 nucleons per $\mathrm{fm}^{3}$. The calculations were performed using the zone determinant method [13] using the second order approximation with zones consisting of a single spatial point. By calculating the exact determinants of some generated matrix configurations we estimate the systemic error for the zone expansion to be about $<0.1 \%$ for $T^{\text {phys }}=37.5 \mathrm{MeV}$ and $<0.5 \%$ for $T^{\text {phys }}=25.0 \mathrm{MeV}$.

We have dealt with the complex action by computing the phase as an observable, and for the various simulations presented here we found an average phase of about $\sim 0.95$ and so this did not present a significant computational problem.

In Fig. 1 we show the energy per neutron as a function of neutron density. Our results indicate a rather flat function for the energy per neutron as a function of density.

The flatness of our energy per neutron curves at these temperatures are intriguing and hopefully will be checked by others in the near future. The results of 3 also see a flattening of the energy per neutron curve with increasing temperatures. We can also compare with variational calculations 14] and recent quantum Monte Carlo results from [15. They observe a significant flattening of the energy per neutron curve due to iteractions even at zero temperature.

\section{REFERENCES}

1. S. Weinberg, Phys. Lett. B251, 288 (1990).

2. R. Seki, U. van Kolck, and M. J. Savage, Singapore, Singapore: World Scientific (1998) $274 \mathrm{p}$.

3. H. M. Müller, S. E. Koonin, R. Seki, and U. van Kolck, Phys. Rev. C61, 044320 (2000), nucl-th/9910038

4. D. Lee, B. Borasoy, and T. Schafer, Phys. Rev. C70, 014007 (2004), nucl-th/0402072.

5. S. Weinberg, Nucl. Phys. B363, 3 (1991).

6. D. B. Kaplan, M. J. Savage, and M. B. Wise, Nucl. Phys. B478, 629 (1996), nucl-th/9605002.

7. S. R. Beane, P. F. Bedaque, M. J. Savage, and U. van Kolck, Nucl. Phys. A700, 377 (2002), nucl-th/0104030

8. G. P. Lepage, (1997), nucl-th/9706029

9. C. Ordonez, L. Ray, and U. van Kolck, Phys. Rev. C53, 2086 (1996), hep-ph/9511380

10. A. Funk and H. V. von Geramb, (2000), nucl-th/0010058.

11. M. Lüscher, Nucl. Phys. B354, 531 (1991).

12. M. Lüscher, Commun. Math. Phys. 105, 153 (1986).

13. D. J. Lee and I. C. F. Ipsen, Phys. Rev. C68, 064003 (2003), nucl-th/0308052.

14. B. Friedman and V. R. Pandharipande, Nucl. Phys. A361, 502 (1981).

15. J. Carlson, J. Morales, J., V. R. Pandharipande, and D. G. Ravenhall, Phys. Rev. C68, 025802 (2003), nucl-th/0302041 